Carolina Quintanilla-Sánchez, Alejandro Salcido-Montenegro, José Gerardo González-González and René Rodríguez-Gutiérrez*

\title{
Acute cerebrovascular events in severe and nonsevere COVID-19 patients: a systematic review and meta-analysis
}

https://doi.org/10.1515/revneuro-2021-0130

Received September 29, 2021; accepted January 17, 2022;

published online February 10, 2022

\begin{abstract}
The degree to which COVID-19 severity influences the development of acute cerebrovascular events (ACVE) is unknown. Therefore, we aimed to describe the prevalence and risk of ACVE in patients with severe and nonsevere COVID-19. We systematically reviewed MEDLINE, EMBASE, Web of Science, and Scopus and identified observational and interventional studies of patients with COVID-19 allocated by respiratory severity that reported ACVE development. Case reports/series were excluded. The main outcome assessed was the pooled rate of ACVE in patients with severe and nonsevere COVID-19. To determine the risk of ACVE development by COVID-19 severity, a meta-analysis was performed. PROSPERO registration number: CRD42020178905. About 19 of 5758 identified studies were analyzed. From 11,886 COVID-19 patients analyzed, 421 had at least one ACVE [3.6\%, 95\% confidence interval (CI) 2.904-4.179]. Severe COVID-19 increased the risk of ACVE (odds ratio 1.96, 95\% CI 1.22-3.15; $P=0.005$;
\end{abstract}

Carolina Quintanilla-Sánchez and Alejandro Salcido-Montenegro contributed equally to this article.

*Corresponding author: René Rodríguez-Gutiérrez, Department of Internal Medicine, University Hospital "Dr. José E. González", Universidad Autónoma de Nuevo León, Monterrey, Ave. Madero y Gonzalitos s/n 64460, México,

E-mail: rodriguezgutierrez.rene@mayo.edu. https://orcid.org/00000002-3022-5302

Carolina Quintanilla-Sánchez, IMPRS Neuroscience, George August Göttingen University, Göttingen 37077, Germany; and Plataforma INVEST UANL-KER Unit Mayo Clinic, School of Medicine and University Hospital “Dr. José E González”, Universidad Autónoma de Nuevo León, Monterrey 64460, México. https://orcid.org/0000-0002-0595-6162 Alejandro Salcido-Montenegro and José Gerardo González-González, Department of Internal Medicine and Plataforma INVEST UANL-KER Unit Mayo Clinic, School of Medicine and "Dr. José E. González" University Hospital, Universidad Autónoma de Nuevo León, Monterrey 64460, N.L. México. https://orcid.org/0000-0002-2487-0241 (A. Salcido-Montenegro). https://orcid.org/0000-0002-6609-9483 (J.G. González-González)
$\left.I^{2}=64 \%\right)$, specifically hemorrhagic stroke (4.12, 2.0-8.53; $\left.P=0.001 ; I^{2}=0 \%\right)$. There was no difference in the risk of developing ischemic stroke between patients with severe and nonsevere COVID-19 (1.53, 0.87-2.7; $\left.P=0.14 ; I^{2}=52 \%\right)$. From the patients who developed any ACVE, those with severe COVID-19 had a greater mortality risk than those with nonsevere COVID-19 (3.85, 1.08-13.70; $P=0.04$; $\left.I^{2}=0 \%\right)$. The main limitations of our study were the heterogeneity found in the main meta-analysis studies and in their reported definition for COVID-19 severity. In conclusion, our findings provide evidence that COVID-19 respiratory severity could lead to ACVE development that increases mortality. The effect of COVID-19 management in ACVE needs to be evaluated.

Keywords: COVID-19; hemorrhagic stroke; ischemic stroke; sinus venous thrombosis; stroke; transitory ischemic attack.

\section{Introduction}

Since the beginning of the pandemic, patients infected with severe acute respiratory syndrome coronavirus 2 (SARS-CoV-2) have presented diverse neurological manifestations, being acute cerebrovascular events (ACVE) among the most important because of their related mortality and impairment of the quality of life (García-Moncó et al. 2020; Mao et al. 2020; Rifino et al. 2020; Romero-Sánchez et al. 2020; Studart-Neto et al. 2020). Both observational and secondary studies have reported a nonnegligible incidence of cerebrovascular complications among patients with COVID-19 (1.1-9.8\%) (Madani Neishaboori et al. 2020; Siepmann et al. 2020; Siow et al. 2021; Yamakawa et al. 2020). The influence that COVID-19 severity has on the risk of presenting ACVE remains uncertain (Altable and de la Serna 2020; Madani Neishaboori et al. 2020; Siepmann et al. 2020; Yamakawa et al. 2020). Disclosing this could elucidate the role that COVID-19 has in the development of ACVE.

Hence, this systematic review aimed to describe and estimate the prevalence and risk of ACVE development in 
patients with severe COVID-19 compared to patients with nonsevere disease.

\section{Methods}

\section{Study design}

We performed a systematic review and meta-analysis, following the Preferred Reporting Items for Systematic Reviews and Meta-Analyses guidelines. The protocol was registered and made publicly available with the international prospective register of systematic reviews on the PROSPERO database (CRD42020178905) on April 20, 2020.

\section{Data sources and searches}

An expert librarian conducted a comprehensive search strategy using controlled vocabulary supplemented with keywords in MEDLINE, EMBASE, Web of Science, and Scopus for all available studies from September 2019 (before the first reported case of COVID-19) to October 2020, with no language restrictions. Among the applied search terms were "COVID-19", "Stroke", "Cerebrovascular disease", "Cerebral hemorrhage”, "Ischemic stroke”, "Transient ischemic attack", "Cerebral infarct", and "Venous sinus thrombosis". Different combinations of these terms as well as their associated Medical Subject Headings were used for the complete search strategy (Supplementary material). We also hand-searched the references of identified full-text and review articles for relevant studies.

\section{Study selection}

Observational and interventional studies of patients with COVID-19 that reported the presence of any ACVE were considered eligible. Case reports and case series were dismissed as prevalence comparison cannot be assessed. We only included studies that allocated adult patients by COVID-19 severity. Although most studies specifically defined the COVID-19 severity criteria, an explicit definition was missing in some of them $(n=5)$. Therefore, we classified the patients as having severe or nonsevere COVID-19 based on the reported groups in the results sections of these studies (e.g., ICU vs. non-ICU admission, mechanical ventilation vs. nonmechanical ventilation). The primary outcome was considered to be any ACVE, such as acute ischemic stroke (AIS), transient ischemic attack (TIA), hemorrhagic stroke, and cerebral venous thrombosis (CVT). Studies reporting microhemorrhages were also included.

Two reviewers (CQ-S and AS-M), working independently and in duplicate, screened all retrieved abstracts and titles in a two-step approach. First, a pilot phase with 40 records was carried out to clarify any misunderstanding and ensure adequate comprehension. Afterward, the formal title and abstract screening were carried out. During this phase, we were highly sensitive, and disagreements between reviewers were included in the full-text phase. Later, the full-text phase was also carried independently and in duplicate to assess eligibility. Reasons for noneligibility were documented, and disagreements at this phase were resolved by consensus with a senior reviewer (RR-G). Interrater agreement for the title/abstract screening and the full text were calculated at each phase using the Kappa statistics.

\section{Data extraction and quality assessment}

Using a standardized web-based form, reviewers working independently and in duplicate collected information from each eligible study. For each study, the baseline clinical features of the included population such as age, gender, comorbidities, and laboratory parameters, in addition to information regarding the outcomes of interest was recorded.

To reduce the reporting bias, we contacted the corresponding authors in case any clarification or more information was needed to determine eligibility or to complete analyses. Two emails were sent within 7 days apart. We contacted seven authors for clarification to obtain further data; one replied.

Two reviewers independently and in duplicate (CQ-S and AS-M) assessed the study quality using the Newcastle-Ottawa risk of bias tool and its modified versions, depending on the observational study type. Besides, the overall quality of the evidence of each outcome was assessed using the Grading of Recommendations Assessment, Development, and Evaluation (GRADE). Disagreements were resolved by consensus or by a senior reviewer (RR-G).

\section{Data synthesis and analysis}

A narrative synthesis for the characteristics of the included studies was conducted, including author, study type, population type and the number of patients, diagnosis of COVID-19, and severity classification. Furthermore, the ACVE prevalence and features, time of onset since COVID-19 diagnosis, incidence, severity, and management were also synthetized. Information of demographics of patients, laboratory parameters, mortality, hospital length of stay, intensive care unit (ICU) admission and length of stay, and mechanical ventilation were also described.

The pooled rate of ACVE along with AIS, hemorrhagic stroke, TIA, and CVT were provided with a 95\% confidence interval (CI). COVID-19 severity definitions were pooled and unified into a severe and nonsevere classification to perform a descriptive review and metaanalysis. Odds ratios (ORs) were calculated for severe and nonsevere patients with COVID-19 from the absolute number of patients with ACVE and each described subtype.

We performed a random-effects model meta-analysis (inverse variance) using Review Manager (RevMan) V.5.4.1 (Computer program, The Cochrane Collaboration, 2020). The $\chi^{2}$ test and the $I^{2}$ statistics were used to assess heterogeneity between studies. A $\chi^{2}$ cut-off value of $P<0.10$ and an $I^{2}$ value $>50 \%$ were considered as indicative of considerable heterogeneity not explained by chance. We decided to combine the different observational studies because the setup was very similar in all of them (data extracted from databases and patients consecutively admitted). However, we explored if study design, type of ACVE, type of population, and COVID-19 confirmatory diagnosis and severity classification were causes of heterogeneity. Studies with significantly very low quality were dismissed from the meta-analysis. The studies that evaluated patients with a confirmed as well as possible and probable COVID-19 diagnosis were included in the main meta-analysis. Likewise, the studies that reported patients with COVID-19 who developed ACVE (general population studies) and those in which the ACVE and other neurological manifestations of patients were analyzed (neurological manifestations cohort studies) were also included in the main meta-analysis. However, we decided to make a subanalysis of the studies that evaluated only patients with laboratory- 
confirmed COVID-19 and of the studies that analyzed the cohort of patients with COVID-19 and other neurological manifestations.

\section{Results}

A total of 5758 studies were retrieved from the electronic databases. After duplicates and noneligible studies were excluded (Figure 1), we selected 19 studies, and 16 of them were included in the meta-analysis (Table 1) (García-Moncó et al. 2020; Kamal et al. 2020; Koh et al. 2020; Kremer et al. 2020; Lachant et al. 2020; Li et al. 2020; Liotta et al. 2020; Lodigiani et al. 2020; Makda et al. 2020; Paterson et al. 2020; Rifino et al. 2020; Romero-Sánchez et al. 2020; Shahjouei et al. 2020; Siepmann et al. 2020; Studart-Neto et al. 2020; Xiao et al. 2020). A total of 11,886 patients with COVID-19 were analyzed. The main reasons for exclusion from the meta-analysis were study design, specific inclusion criteria for the studied population, and lack of information about the COVID-19 and stroke diagnosis criteria.

The reviewed studies included cross-sectional $(n=4)$, case-controlled ( $n=1)$, observational prospective $(n=2)$, retrospective $(n=11)$, and ambispective studies $(n=1)$. The

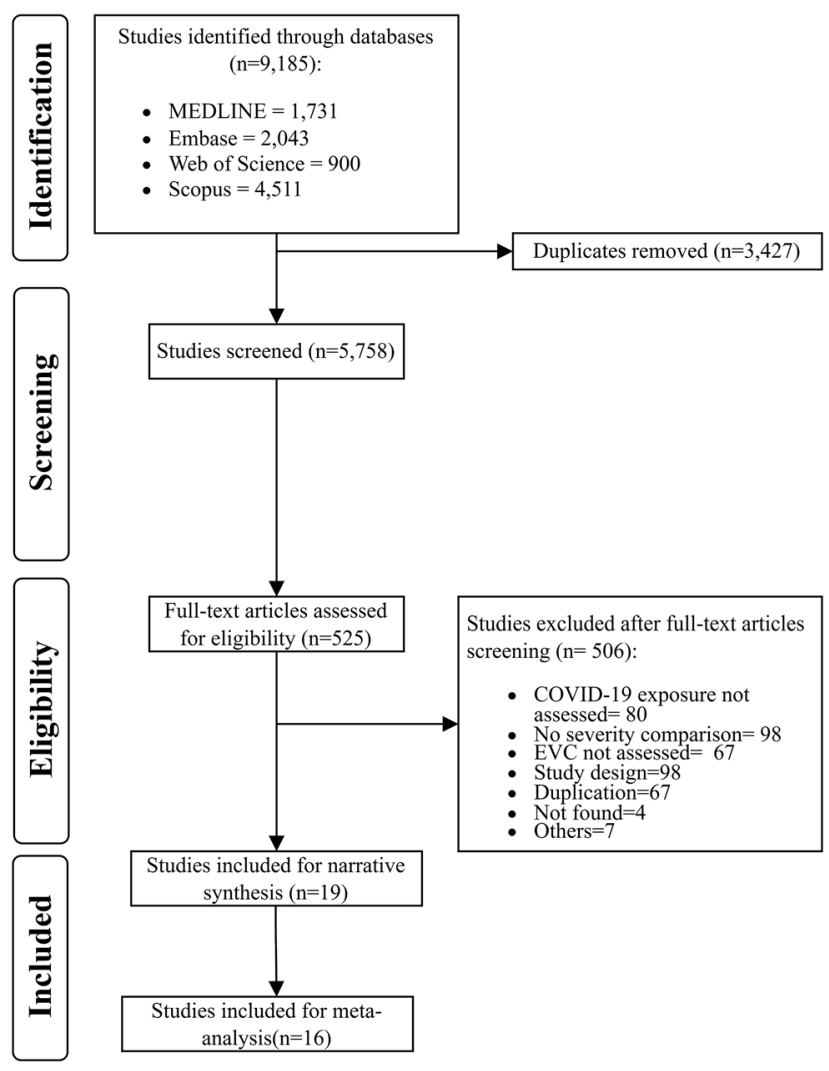

Figure 1: Flow diagram showing the identification of eligible and included studies.
COVID-19 diagnosis was made with SARS-CoV-2 real-time polymerase chain reaction alone $(n=10)$ or with serology $(n=3)$, as well as based on clinical and laboratory features highly suggestive of COVID-19 and/or compatible radiologic findings $(n=6)$. Seven studies included only patients with COVID-19 who developed ACVE and/or other neurological manifestations. The COVID-19 severity was defined by the necessity of ICU admission $(n=5)$, mechanical ventilation $(n=5)$, diverse pneumonia severity criteria $(n=3)$, presence of acute respiratory distress syndrome (ARDS; $n=2$ ), or by following regional and international guidelines for COVID-19 management ( $n=4$; Table 1).

\section{Quality of evidence}

All but one study analyzed severity groups from the same population; all studies had exposure and outcome assessment in a very similar manner. However, most studies lacked control of confounding factors for ACVE. Two studies adjusted for age, arterial hypertension, diabetes mellitus (Siepmann et al. 2020), and ischemic heart disease were needed (Shahjouei et al. 2020). In most studies, it was unclear whether the outcome occurred after exposition or how many days after. Besides, follow-up was not explicitly stated. Thus, the overall quality of evidence was poor (Supplementary Table 1).

\section{ACVE prevalence and risk}

Of the 11,886 patients with COVID-19, 421 had an ACVE (females $37.08 \%$, mean range of ages: $51.5-77$ years) with an overall rate of 3.6\% (95\% CI 2.904-4.179) (Supplementary Tables 2 and 3). Only 7 of the 19 analyzed studies described the treatment used for the management of ACVE (Supplementary Table 3).

The meta-analysis showed that severe COVID-19 increased the risk for ACVE (OR 1.96, 95\% CI 1.22-3.15; $P=0.005)\left(I^{2}=64 \%\right.$; Figure 2). The GRADE evaluation for the increased risk of ACVE in severe COVID-19 was low due to (1) all studies being observational, (2) most studies not controlling for known ACVE risk factors, (3) follow-up periods not described, and (4) significant heterogeneity (which decreased in subanalyses; Supplementary Table 4). The overall rate of AIS, hemorrhagic stroke, CVT, and TIA was $2.776 \%$ ( $n=327 / 11,779,95 \%$ CI $2.274-3.277)$, $0.859 \%$ ( $n=77 / 8,963,95 \%$ CI 0.662-1.056), 0.207\% ( $n=14 / 6,745$, 95\% CI $0.155-0.259)$, and 2.551\% ( $n=10 / 392,95 \%$ CI 2.493-2.607), respectively (Supplementary Table 3). 


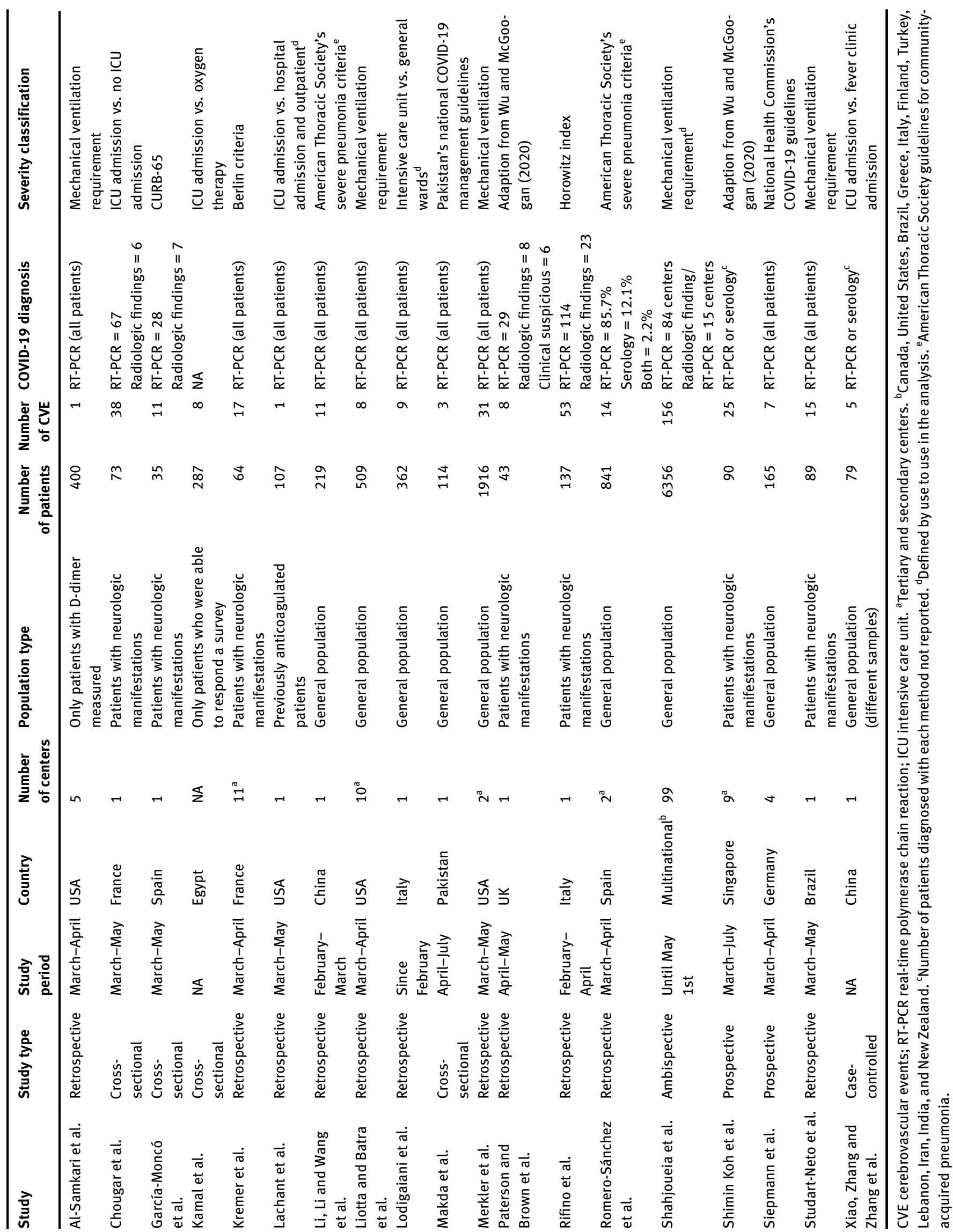




\section{(A)}

\begin{tabular}{|c|c|c|c|c|c|}
\hline & Severe & Exp & Control - No & vere & \\
\hline Study or Subgroup & Events & Total & Events & Total & Weight \\
\hline Al-Samkari et al. & 1 & 144 & 0 & 256 & $1.8 \%$ \\
\hline Chougar et al. & 23 & 35 & 15 & 38 & $7.9 \%$ \\
\hline García-Moncó et al. & 5 & 13 & 6 & 22 & $5.5 \%$ \\
\hline Kremer et al. & 5 & 33 & 14 & 31 & $6.8 \%$ \\
\hline Li, Li \& Wang et al. & 9 & 92 & 2 & 127 & $5.2 \%$ \\
\hline Liotta \& Batra et al. & 6 & 134 & 2 & 375 & $5.0 \%$ \\
\hline Lodigaiani et al. & 3 & 48 & 6 & 314 & $5.7 \%$ \\
\hline Makda et al. & 3 & 62 & 0 & 52 & $2.1 \%$ \\
\hline Merkler et al. & 11 & 330 & 20 & 1586 & $9.1 \%$ \\
\hline Peterson \& Brown et al. & 7 & 17 & 6 & 26 & $6.1 \%$ \\
\hline Rifino et al. & 22 & 69 & 31 & 68 & $9.4 \%$ \\
\hline Romero-Sánchez et al. & 7 & 329 & $?$ & 512 & $7.4 \%$ \\
\hline Shahjoueia et al. & 31 & 469 & 125 & 5887 & $10.8 \%$ \\
\hline Shimin Koh et al. & 5 & 14 & 20 & 76 & $6.7 \%$ \\
\hline Siepmann et al. & 6 & 119 & 1 & 46 & $3.4 \%$ \\
\hline Studart-Neto et al. & 8 & 45 & 7 & 44 & $7.1 \%$ \\
\hline Total $(95 \% \mathrm{Cl})$ & & 1953 & & 9460 & $100.0 \%$ \\
\hline Total events & 152 & & 262 & & \\
\hline $\begin{array}{l}\text { Heterogeneity: Tau }=0 \\
\text { Test for overall effect: } Z\end{array}$ & $\begin{array}{l}\mathrm{Chi}^{2}=4 \\
.79\langle\mathrm{P}=\end{array}$ & & $5(P=0.0$ & $1-04$ & \\
\hline
\end{tabular}

Odds Ratio

Random, $95 \% \mathrm{Cl}$

$5.36[0.22,132.49]$

$2.94[1.13,7.63]$

$1.67[0.39,7.17]$

$0.22[0.07,0.71]$

$6.78[1.43,32.16]$

$8.74[1.74,43.86]$

$3.42[0.83,14.17]$

$6.18[0.31,122.37]$

$2.70[1.28,5.69]$

$2.33[0.62,8.81]$

$0.56[0.28,1.12]$

$1.57[0.55,4.51]$

$3.26[2.18,4.89]$

$1.56[0.43,5.20]$

$2.39[0.28,20.41]$

$1.14[0.38,3.47]$

$1.96[1.22,3.15]$
Odds Ratio IN, Random, $95 \% \mathrm{Cl}$

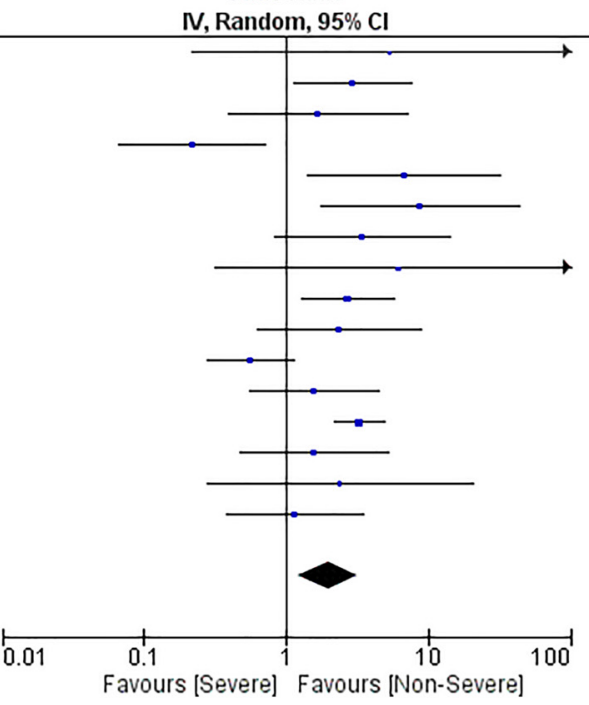

(B)

Study or Subgroup

Chougar et al.

García-Moncó et al.

Kremer et al.

$\mathrm{Li}, \mathrm{Li} \&$ Wang et al.

Liotta \& Batra et al.

Lodigaiani et al.

Makda et al.

Merkler et al.

Peterson \& Brown et al.

Romero-Sánchez et al.

Shimin Koh et al.

Siepmann et al.

Studart-Neto et al.

Total ( $95 \% \mathrm{Cl})$

Total events

Heterogeneity: $\mathrm{Tau}^{2}=0.53 ; \mathrm{Chi}^{2}=25.24, \mathrm{df}=12(\mathrm{P}=0.01) ; \mathrm{I}^{2}=52 \%$

Test for overall effect: $Z=1.48(P=0.14)$
Severe Non-severe Odds Ratio Events Total Events Total Weight M-H, Random, 95\% Cl

$\begin{array}{rrrrr}9 & 35 & 8 & 38 & 10.0 \% \\ 4 & 13 & 6 & 22 & 7.5 \% \\ 3 & 33 & 14 & 31 & 8.1 \% \\ 8 & 92 & 2 & 127 & 7.1 \% \\ 5 & 134 & 2 & 375 & 6.7 \% \\ 3 & 48 & 6 & 314 & 7.9 \% \\ 3 & 62 & 0 & 52 & 2.9 \% \\ 11 & 330 & 20 & 1586 & 12.4 \% \\ 3 & 17 & 5 & 26 & 7.1 \% \\ 4 & 329 & 7 & 512 & 9.0 \% \\ 3 & 14 & 13 & 76 & 8.0 \% \\ 3 & 119 & 1 & 46 & 4.4 \% \\ 6 & 45 & 5 & 44 & 8.8 \%\end{array}$

1271

$3249 \quad 100.0 \%$ 89
$1.30[0.44,3.85]$
$1.19[0.26,5.34]$
$0.12[0.03,0.48]$
$5.95[1.23,28.72]$
$7.23[1.39,37.71]$
$3.42[0.83,14.17]$
$6.18[0.31,122.37]$
$2.70[1.28,5.69]$
$0.90[0.18,4.38]$
$0.89[0.26,3.06]$
$1.32[0.32,5.41]$
$1.16[0.12,11.48]$
$1.20[0.34,4.26]$

$\mathbf{1 . 5 3}[0.87,2.70]$
(C)

Severe Non-Severe Odds Ratio Study or Subgroup Events Total Events Total Weight IN, Random, 95\% Cl

\begin{tabular}{|c|c|c|c|c|c|}
\hline Al-Samkari et al. & 1 & 144 & 0 & 256 & $5.8 \%$ \\
\hline Chougar et al. & 13 & 35 & 7 & 38 & $51.8 \%$ \\
\hline García-Moncó et al. & 1 & 13 & 0 & 22 & $5.5 \%$ \\
\hline Kremer et al. & 2 & 33 & 0 & 31 & $6.3 \%$ \\
\hline Li, Li \& Wang et al. & 1 & 92 & 0 & 127 & \\
\hline Liotta \& Batra et al. & 1 & 134 & 0 & 375 & $5.8 \%$ \\
\hline Peterson \& Brown et al. & 4 & 17 & 1 & 26 & $11.3 \%$ \\
\hline Romero-Sánchez et al. & 3 & 329 & 0 & 512 & \\
\hline Shimin Koh et al. & 2 & 14 & 0 & 76 & $6.2 \%$ \\
\hline Studart-Neto et al. & 1 & 45 & 1 & 44 & $7.5 \%$ \\
\hline Total $(95 \% \mathrm{Cl})$ & \multicolumn{3}{|c|}{435} & 868 & $100.0 \%$ \\
\hline Total events & \multirow{2}{*}{\multicolumn{5}{|c|}{$\begin{array}{l}25 \\
i^{2}=3.83, d f=\end{array}$}} \\
\hline Heterogeneity: $\operatorname{Tau}^{2}=0.0$ & & & & & \\
\hline
\end{tabular}

[0.22,132.49]

$2.62[0.90,7.62]$

$5.40[0.20,142.71]$

$5.00[0.23,108.39]$

Not estimable

$8.44[0.34,208.40]$

$7.69[0.78,76.08]$

Not estimable

$0.60[1.39,675.65]$

$0.98[0.06,16.13]$

$3.86[1.79,8.33]$

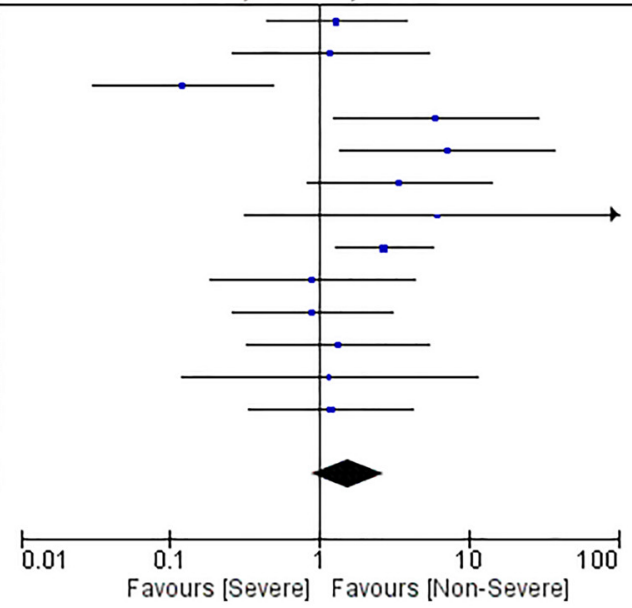
M-H, Random, $95 \% \mathrm{Cl}$

Odds Ratio

$\longrightarrow \cdot$


The overall rate of ACVE was 7.951\% ( $n=164 / 2050$, 95\% CI 7.602-8.299) for patients with severe COVID-19 and $2.69 \%$ ( $n=265 / 9836,95 \%$ CI $2.136-3.251)$ for those with nonsevere COVID-19. Patients with severe COVID-19 had an increased risk of presenting hemorrhagic stroke (OR 4.12, 95\% CI 2.0-8.53; $P=0.001 ; I^{2}=0 \%$ ), but not AIS (OR 1.53, 95\% CI 0.87-2.7; $P=0.14 ; I^{2}=52 \%$ ), CVT (OR 1.15 , $95 \%$ CI $0.20-6.45 ; P=0.88 ; I^{2}=0 \%$ ), or TIA (OR $1.43,95 \%$ CI $0.17-11.95 ; P=0.53 ; I^{2}=0 \%$ ) (Figure 2 and Supplementary Figure 1).

\section{General population}

From the 11,355 patients with CsOVID-19 pooled from the general population studies, 254 patients had ACVE (2.236\%, 95\% CI 1.442-3.031). Severe COVID-19 increases the risk for ACVE (OR 3.19, 95\% CI 2.34-4.34; $P=0.000001$; $I^{2}=0 \%$ ), AIS (OR 2.73, 95\% CI 1.61-4.65; $P=0.0002$; $I^{2}=6 \%$ ), and hemorrhagic stroke (OR 6.89, 95\% CI 1.4333.15; $P=0.02 ; I^{2}=0 \%$ ) (Supplementary Figure 2).

\section{Neurological manifestations cohort}

From the 531 patients with COVID-19 pooled from the cohort studies of neurological manifestations, 174 had an ACVE (33.768\%, 95\% CI 31.455-34.080) (Supplementary Figure 3). When analyzing the cohort studies of neurological manifestations, severe COVID-19 patients presented higher risk of hemorrhagic stroke (OR 3.59, 95\% CI 1.588.13; $P=0.002 ; I^{2}=0 \%$ ), but not for overall ACVE (OR 1.10 , 95\% CI 0.55-2.19; $P=0.78 ; I^{2}=64 \%$ ) or AIS (OR 0.82, 95\% CI 0.39-1.72; $P=0.59 ; I^{2}=45 \%$ ) (Supplementary Figure 3).

\section{Mortality}

The overall mortality rate in patients with COVID-19 and ACVE was 29.92\% ( $n=41 / 137,95 \%$ CI 29.808-30.045), with severe COVID-19 and ACVE conferring a greater mortality risk (OR 3.85, 95\% CI 1.08-13.70; $P=0.04 ; I^{2}=0 \%$ ) (Figure 3). The GRADE evaluation was moderate in this regard due to (1) study design, (2) lack of confounding factors control for mortality, (3) time to event was not reported, but (4) magnitude of the effect was importantly high (Supplementary Table 5).

\section{Laboratory-confirmed COVID-19}

From the 4769 patients with laboratory-confirmed COVID-19, 143 patients developed ACVE (2.998\%, 95\% CI 2.739-3.257). In the analysis of the laboratory-confirmed COVID-19 studies, severe COVID-19 patients presented an increased risk of developing any ACVE (OR 2.08, 95\% CI 1.10-3.91; $P=0.02 ; I^{2}=55 \%$ ) and hemorrhagic stroke (OR 5.81, 95\% CI 1.82-18.54; $\left.P=0.003 ; I^{2}=0 \%\right)$. There was no higher risk for AIS (OR 1.71, 95\% CI 0.81-3.58; $P=0.16$; $I^{2}=63 \%$ ) (Supplementary Figures 4-6).

\section{Discussion}

In this systematic review and meta-analysis, we found an increased risk of ACEs in patients with severe COVID-19 in comparison with nonsevere cases. This association remained in the subanalyses of the general population and laboratoryconfirmed COVID-19 studies. Nevertheless, in the cohort studies of neurological manifestations, we did not observe a higher risk for ACVE. Two studies could have contributed to this outcome, in which authors reported a major proportion of AIS in patients with nonsevere COVID-19. Both studies defined severity with ARDS-related criteria (Kremer et al. 2020; Rifino et al. 2020). A more rigorous severity classification could include more patients under mechanical ventilation, in whom ACVE could be underdiagnosed (Siepmann et al. 2020). Nevertheless, in most studies using the need for mechanical ventilation as severity criteria,

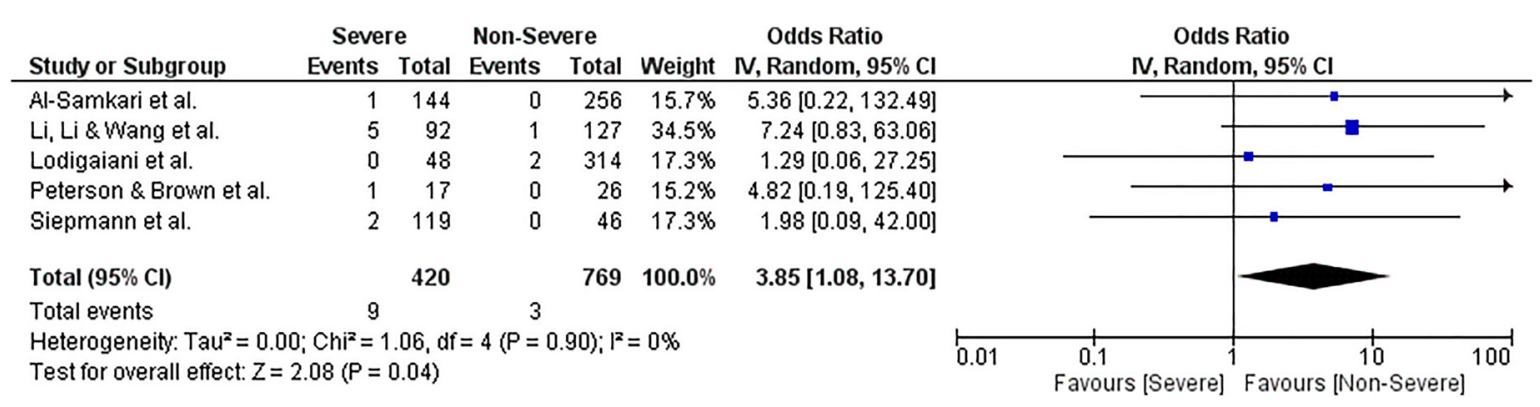

Figure 3: Mortality risk in ACVE by COVID-19 severity. 
patients with severe COVID-19 were at higher risk for any ACVE. COVID-19-specific severity criteria is more balanced and less prone to misclassify respiratory severity (Xiao et al. 2020).

In the subanalyses by ACVE type, there was no suggestion of an increased risk for AIS in patients with severe COVID-19. However, when the cohort studies of neurological manifestations were excluded from the analysis, severe COVID-19 presented a higher risk of AIS in the general population (in both laboratory-confirmed and possible/probable COVID-19 diagnosis study subanalyses). This contrasts with the current published data (Siepmann et al. 2020). The systematic review and meta-analysis by Siepmann and colleagues concluded that patients with severe COVID-19 have a higher risk of AIS. Nonetheless, only two studies were analyzed (Siepmann et al. 2020).

In our analysis, Kremer et al. reported the highest rate of AIS in nonsevere COVID-19 patients and proposed that the procoagulant state of the disease could explain the AIS among other thrombotic events (Kremer et al. 2020). However, in most of the analyzed studies in our review, patients had been anticoagulated as early as they were diagnosed with COVID-19. Apart from a hypercoagulability state, three other possible mechanisms had been proposed to explain the observed AIS: the alternative renin-angiotensin system pathway, cardioembolism and COVID-19-associated cardiopathy, and SARS-CoV-2 damage of the neurovascular unit. Studies controlling anticoagulation as a confounding factor are needed.

A higher proportion of brain hemorrhages and microhemorrhages were reported in the studies evaluating COVID-19 patients with other neurological manifestations who underwent brain magnetic resonance image (MRI). Chougar et al. reported that almost one-third of the patients had microhemorrhages in the brain MRI. The authors suggested that ECMO and anticoagulation overdose could explain some of the cases. Nevertheless, 17 cases were speculated to be related to SARS-CoV-2 infection (Chougar et al. 2020). Kremer et al. and Paterson et al. also reported multiple brain hemorrhagic lesions observed in MRI, but in the context of acute disseminated encephalomyelitis (ADEM) (Kremer et al. 2020; Paterson et al. 2020). Described as a rare autoimmune disease occurring after viral infections and vaccinations leading to a rapid onset of neurological deficits, various cases of ADEM and hemorrhagic necrotizing encephalopathy have been reported in patients with COVID-19 (Correia et al. 2020; Sonneville et al. 2009). The fact that only studies that evaluated brain MRI reported these cases suggests that microhemorrhages and encephalopathy-related brain hemorrhages could be underdiagnosed complications of COVID-19.

Our results evidence that severe COVID-19 increases the risk for ACVE, expanding the outcomes of previous reviews. Yink-Kiat et al. analyzed 4466 COVID-19 patients from 39 studies, describing an AIS rate of $1.2 \%$ and a mortality rate of 38\% (Tan et al. 2020). Yamakawa et al. analyzed 6368 COVID-19 patients and described an ACVE rate of $1.1 \%$ (AIS $96.6 \%$, hemorrhagic 3.4\%) and a mortality rate of 44.2\% (Yamakawa et al. 2020). Both studies had less proportion of AIS and a higher mortality rate compared to our study. Madant Nelshaboorl et al. analyzed 1634 COVID-19 patients and reported an ACVE rate of 2.59\%, similar to our study (Madani Neishaboori et al. 2020).The systematic review and meta-analysis by Siow et al. reported an incidence of 9.8\% ACVE (2.7-30.6\%) and a high mortality rate $(84.8 \%)$ in patients with severe COVID-19 (Siow et al. 2021). Yet, the low to very low quality of the analyzed studies and the inclusion of case reports and series could hinder these results.

Siepmann et al. reported an overall ACVE rate of 2.9\% as well as an increased risk ratio of AIS in patients with severe COVID-19 (4.18, 95\% CI 1.7-10.25; $P=0.002$ ) (Siepmann et al. 2020). As mentioned before, this contrasted with the lack of risk for severe COVID-19 of presenting AIS in most of our analyses. Similarly, a recent systematic review and meta-summary by $\mathrm{Lu}$ et al. described a rate of $1.76 \%$ of AIS as well as an increased risk in severe COVID-19 (RR = 3.53, 95\% CI 2.06-6.07; $P<0.001)$ (Lu et al. 2021).

Although valuable data, results by Siepmann et al. and Lu et al. were limited by the number of studies analyzed, lack of quality assessment, and the absence of other ACVE subtypes (Siepmann et al. 2020; Lu et al. 2021).

A great extent of the emerging COVID-19 studies had been of low-moderate quality. Unsurprisingly, we did not find any clinical trial assessing ACVE in severe and nonsevere COVID-19 cases, and the majority of the found studies have major concerns about cofounding factors, event onset, and follow-up. Only two of the analyzed studies accounted for the known risk factors of ACVE when comparing the severe and nonsevere COVID-19 population. The multivariable analysis by Siepmann et al. showed that the need for intensive care was an independent factor for AIS (OR 4.69, 95\% CI 1.0-21.9) (Siepmann et al. 2020). The binary logistic regression by Shahjoueia et al. suggested mechanical ventilation as an independent predictor of ACVE (OR 1.9, $95 \%$ CI 1.1-3.4). To assess if there is any true effect, higher quality studies are needed (Shahjouei et al. 2020). 
In the context of severe COVID-19, clinicians must be alert for the development of ACVE as a possible complication, especially when neurological signs such as focalization cannot be easily assessed (e.g., patients on mechanical ventilation).

Our study had several limitations. First, the study heterogeneity found in the main meta-analysis. Nevertheless, when the cohort studies of neurological manifestations were separated from the rest, study heterogeneity diminished considerably. Apart from only including patients with COVID-19 and neurological manifestations, some studies included patients with brain MRI or who were referred to a neurology center exclusively. We believed these were important factors contributing to heterogeneity among studies. Second, the definition for COVID-19 was heterogeneous and, in some cases, it was not specified by the authors. Nevertheless, when this was considered in sensitivity analysis, it did not seem to alter the obtained results. As mentioned above, the inclusion criteria seemed to contribute more to the heterogeneity study than the diverse COVID-19 severity definitions used. Finally, studies that evaluated patients with confirmed and suspected COVID-19 diagnoses were included. Because of potential false-negative results, we decided to include these studies, resonating more with the current state of the pandemic. However, the results of the subanalysis of the studies including only patients with confirmed COVID-19 diagnosis were no different from the overall meta-analysis.

\section{Conclusions}

Our findings provide evidence that COVID-19 respiratory severity increases the risk of ACVE development, which furtherly increases mortality. Physicians must acknowledge ACVE as a possible complication of patients with severe COVID-19. Higher quality observational and interventional studies are needed to disclose the true effect of COVID-19 over ACVE development and the effects that current and future treatments have on this outcome.

Acknowledgments: We acknowledge the librarian NAV for conducting the search strategy.

Author contributions: CQ-S and AS-M designed the study, selected the articles, assessed the quality of the studies, extracted data, conducted the analysis, and wrote the first draft. JGG-G and RR-G contributed to the interpretation of results and the final manuscript.
Research funding: None declared.

Conflict of interest statement: The authors declared that they have no conflict of interest.

\section{References}

Altable, M. and de la Serna, J.M. (2020). Cerebrovascular disease in COVID-19: is there a higher risk of stroke?. Brain Behav. Immun. Health 6: 100092.

Chougar, L., Shor, N., Weiss, N., Galanaud, D., Leclercq, D., Mathon, B., and Belkacem, S. (2020). Retrospective observational study of brain magnetic resonance imaging findings in patients with acute SARS-CoV-2 infection and neurological manifestations. Radiology 297: E313-E323.

Correia, A.O., Feitosa, P.W.G., Moreira, J.L.de S., Nogueira, S.Á.R., Fonseca, R.B., and Nobre, M.E.P. (2020). Neurological manifestations of COVID-19 and other coronaviruses: a systematic review. Neurol. Psychiatry Brain Res. 37: 27-32.

García-Moncó, J.C., Cabrera-Muras, A., Collía-Fernández, A., ErburuIriarte, M., Rodrigo-Armenteros, P., Oyarzun-Irazu, I., MartínezCondor, D., Bilbao-González, A., Carmona-Abellán, M., Caballero-Romero, I., et al. (2020). Neurological reasons for consultation and hospitalization during the COVID-19 pandemic. Neurol. Sci. 41: 3031-3038.

Kamal, M., Abo Omirah, M., Hussein, A., and Saeed, H. (2020). Assessment and characterisation of post-COVID-19 manifestations. Int. J. Clin. Pract. 75: e13746.

Koh, J.S., Silva, D.A. De, May, A., Quek, L., Chiew, H.J., Ming, T., Ying, C., Seet, H., Hui, R., Hoe, M., et al. (2020). Neurology of COVID-19 in Singapore. J. Neurol. Sci. 418: 117118.

Kremer, S., Lersy, F., Anheim, M., Merdji, H., Schenck, M., Oesterlé, H., Bolognini, F., Messie, J., Khalil, A., Gaudemer, A., et al. (2020). Neurologic and neuroimaging findings in patients with COVID-19: a retrospective multicenter study. Neurology 95: e1868-e1882.

Lachant, D.J., Lachant, N.A., Kouides, P., Rappaport, S., Prasad, P., and White, R.J. (2020). Chronic therapeutic anticoagulation is associated with decreased thrombotic complications in SARS-CoV-2 infection. J. Thromb. Haemost. 18: 2640-2645.

Li, Y., Li, M., Wang, M., Zhou, Y., Chang, J., Xian, Y., Wang, D., Mao, L., Jin, H., and Hu, B. (2020). Acute cerebrovascular disease following COVID-19: a single center, retrospective, observational study. Stroke Vasc. Neurol. 5: 279-284.

Liotta, E.M., Batra, A., Clark, J.R., Shlobin, N.A., Hoffman, S.C., Orban, Z.S., and Koralnik, I.J. (2020). Frequent neurologic manifestations and encephalopathy-associated morbidity in Covid-19 patients. Ann. Clin. Transl. Neurol. 7: 2221-2230.

Lodigiani, C., Iapichino, G., Carenzo, L., Cecconi, M., Ferrazzi, P., Sebastian, T., Kucher, N., Studt, J.D., Sacco, C., Alexia, B., et al. (2020). Venous and arterial thromboembolic complications in COVID-19 patients admitted to an academic hospital in Milan, Italy. Thromb. Res. 191: 9-14.

Lu, Y., Zhao, J., Ye, M.-F., fan, M., Li, H.-M., Yao, F.-R., Kong, Y., and Xu, Z. (2021). The relationship between COVID-19's severity and ischemic stroke: a systematic review and meta-analysis. Neurol. Sci. 42: 2645-2651. 
Madani Neishaboori, A., Moshrefiaraghi, D., Mohamed Ali, K., Toloui, A., Yousefifard, M., and Hosseini, M. (2020). Central nervous system complications in COVID-19 patients; a systematic review and meta-analysis based on current evidence. Arch. Acad. Emerg. Med. 8: e62.

Makda, A., Kumar, S., Kumar, A., Kumar, V., and Rizwan, A. (2020). The frequency of neurological symptoms in COVID-19 patients at a tertiary care hospital in Pakistan. Cureus 12: 12-16.

Mao, L., Jin, H., Wang, M., Hu, Y., Chen, S., He, Q., Chang, J., Hong, C., Zhou, Y., Wang, D., et al. (2020). Neurologic manifestations of hospitalized patients with coronavirus disease 2019 in Wuhan, China. JAMA Neurol. 77: 683-690.

Paterson, R.W., Brown, R.L., Benjamin, L., Nortley, R., Wiethoff, S., Bharucha, T., Jayaseelan, D.L., Kumar, G., Raftopoulos, R.E., Zambreanu, L., et al. (2020). The emerging spectrum of COVID-19 neurology: clinical, radiological and laboratory findings. Brain 143: 3104-3120.

Rifino, N., Censori, B., Agazzi, E., Alimonti, D., Bonito, V., Camera, G., Conti, M.Z., Foresti, C., Frigeni, B., Gerevini, S., et al. (2020). Neurologic manifestations in 1760 COVID-19 patients admitted to Papa Giovanni XXIII Hospital, Bergamo, Italy. J. Neurol. 268: 2331-2338.

Romero-Sánchez, C.M., Díaz-Maroto, I., Fernández-Díaz, E., SánchezLarsen, Á., Layos-Romero, A., García-García, J., González, E., Redondo-Peñas, I., Perona-Moratalla, A.B., Del Valle-Pérez, J.A., et al. (2020). Neurologic manifestations in hospitalized patients with COVID-19: the ALBACOVID registry. Neurology 95: e1060-e1070.

Shahjouei, S., Naderi, S., Li, J., Khan, A., Chaudhary, D., Farahmand, G., Male, S., Griessenauer, C., Sabra, M., Mondello, S., et al. (2020). Risk of stroke in hospitalized SARS-CoV-2 infected patients: a multinational study. EBioMedicine 59: 102939.
Siepmann, T., Sedghi, A., Simon, E., Winzer, S., Barlinn, J., de With, K., Mirow, L., Wolz, M., Gruenewald, T., Schroettner, P., et al. (2020). Increased risk of acute stroke among patients with severe COVID-19: a multicenter study and meta-analysis. Eur. J. Neurol. 28: 238-247.

Siow, I., Lee, K.S., Zhang, J.J.Y., Saffari, S.E., Ng, A., and Young, B. (2021). Stroke as a neurological complication of COVID-19: a systematic review and meta-analysis of incidence, outcomes and predictors. J. Stroke Cerebrovasc. Dis. 30: 105549.

Sonneville, R., Klein, I., de Broucker, T., and Wolff, M. (2009). Postinfectious encephalitis in adults: diagnosis and management. J. Infect. 58: 321-328.

Studart-Neto, A., Guedes, B.F., de Luca e Tuma, R., Camelo Filho, A.E., Kubota, G.T., lepsen, B.D., Moreira, G.P., Rodrigues, J.C., Ferrari, M.M.H., Carra, R.B., et al. (2020). Neurological consultations and diagnoses in a large, dedicated COVID-19 university hospital. Arq. Neuropsiquiatr. 78: 494-500.

Tan, Y.K., Goh, C., Leow, A.S.T., Tambyah, P.A., Ang, A., Yap, E.S., Tu, T.M., Sharma, V.K., Yeo, L.L.L., Chan, B.P.L., et al. (2020). COVID-19 and ischemic stroke: a systematic review and meta-summary of the literature. J. Thromb. Thrombolysis 50: 587-595.

Xiao, M., Zhang, Y., Zhang, S., Qin, X., Xia, P., Cao, W., Jiang, W., Chen, H., Ding, X., Zhao, H., et al. (2020). Antiphospholipid antibodies in critically ill patients with COVID-19. Arthritis Rheumatol. 72: 1998-2004.

Yamakawa, M., Kuno, T., and Mikami, T. (2020). Clinical characteristics of stroke with COVID-19: a systematic review and meta-analysis. J. Stroke Cerebrovasc. Dis. 29: 105288.

Supplementary Material: The online version of this article offers the supplementary material (https://doi.org/10.1515/revneuro-2021-0130). 